- RAHIS, Revista de Administração Hospitalat e Inovação em Saúde Vol. I6, n4 " Belo Horizonte, MG = OUT/DEZ 20I9 "e-ISSN: 2177 2754 e ISSN impresso: I983-5205 " DOI: https://doiorg/I0.21450/rahis.vI6i4.59I7 " Submetido: (08/I2/20I9) " Aceito: (18/05/2020)" Sistema de avaliação: Double Blind Review "p. $63-80$

EFEITO DA RESPONSABILIDADE SOCIAL CORPORATIVA E DA GOVERNANÇA CORPORATIVA NO DESEMPENHO EMPRESARIAL NO SETOR DE SAÚDE

\title{
EFFECT OF CORPORATE SOCIAL RESPONSIBILITY AND CORPORATE
} GOVERNANCE ON CORPORATE PERFORMANCE IN THE HEALTH CARE SECTOR

EFECTO DE LA RESPONSABILIDAD SOCIAL CORPORATIVA Y DE LA GOBERNANZA CORPORATIVA EN EL DESEMPEÑO EMPRESARIAL EN EL SECTOR DE SALUD

Jessica Mendes De Luca Centro Universitário Christus - Unichristus jessicamdeluca@hotmail.com

Alan Diógenes Góis alandgois@hotmail.com

Marcia Martins Mendes De Luca Universidade Federal do Ceará marciammdeluca@gmail.com

Jislene Trindade Medeiros Universidade Federal do Ceará jislenetm@gmail.com 


\section{RESUMO}

O estudo tem o objetivo de analisar o efeito da responsabilidade social corporativa e da governança corporativa no desempenho empresarial no setor de saúde. Para tanto, por meio de regressão quantílica com dados em painel, são analisadas 453 empresas de capital aberto (I.825 observações) do setor de saúde, de 3 I países, que possuem dados nas bases Thomson Reuters Eikon e World Bank, referentes ao período 20I0-20I8. Os resultados demonstram que a responsabilidade social corporativa e a governança corporativa são positivamente correlacionadas. Verificou-se que as práticas de responsabilidade social corporativa e a governança corporativa afetam positivamente o desempenho empresarial de mercado, mensurado pelo Q de Tobin, e negativamente o desempenho empresarial operacional, medido pelo Return On Assets. Desse modo, verificase que as práticas ambientais, sociais e de governança corporativa são relevantes para a legitimação das empresas do setor de saúde apenas na perspectiva da criação de valor. Portanto, considerando-se a alta relevância social e econômica do segmento, tem-se que, para se perpetuar no mercado, a empresa do setor de saúde deve buscar melhorias ou implementar práticas de responsabilidade social corporativa e de governança corporativa.

Palavras-chave: Responsabilidade social corporativa. Governança corporativa. Desempenho empresarial.

\section{ABSTRACT}

In this study we evaluated the effect of corporate social responsibility and corporate governance on corporate performance in the health care sector. Information on 453 public firms (I,825 observations) from the health care sector of 3I countries was retrieved from the platforms of Thomson Reuters Eikon and World Bank, covering the period 2010-2018, and submitted to quantile regression on panel data. Corporate social responsibility and corporate governance were found to be positively correlated. Corporate social responsibility practices and corporate governance had a positive effect on market value (Tobin's Q) but a negative effect on corporate operating performance (Return On Assets). In other words, the legitimacy acquired through environmental, social and corporate governance practices benefited firms only from the perspective of value creation. Considering the substantial social and economic importance of the sector, health care organizations looking to remain on the market should invest in improvements or implement corporate social responsibility and corporate governance practices.

Key words: Corporate social responsibility. Corporate governance. Corporate performance.

\section{RESUMEN}

El estudio tiene por objeto analizar el efecto de la responsabilidad social corporativa y de la gobernanza corporativa en el desempeño empresarial del sector salud. Para tanto, por medio de la regresión de cuantiles con datos de panel, son analizadas 453 empresas de capital abierto del sector salud (I.825 observaciones) en 3 I países, que poseen datos en las bases Thomson Reuters Eikon y World Bank, referentes al período 20I0-2018. Los resultados demuestran que la responsabilidad social corporativa y la gobernanza corporativa están positivamente correlacionadas. Las practicas de responsabilidad social corporativa y la gobernanza corporativa afectan favorablemente el desempeño empresarial de mercado, medido por la $Q$ de Tobin, y negativamente el desempeño empresarial operacional, medido por el Return On Assets. Así, se verifica que las prácticas ambientales, sociales y de gobernanza corporativa son relevantes para la legitimación de las empresas del sector salud apenas en la creación de valor. Por lo tanto, considerando la alta relevancia social y económica del segmento, vemos que, para perpetuarse en el mercado, la empresa del sector salud debe buscar mejorías o implementar prácticas de responsabilidad social corporativa y de gobernanza corporativa.

Palabras clave: Responsabilidad social corporativa. Gobernanza corporativa. Desempeño empresarial.

\section{INTRODUÇÃO}

No atual contexto de globalização, o setor de serviços possui uma grande representatividade nos indicadores de desenvolvimento econômico das nações. Dentre seus vários segmentos especializados, a saúde tem se destacado pela maior velocidade de crescimento, assim como pelo acelerado aumento da concorrência (LONIAL et al., 2008).

Segundo Jamali, Hallal e Abdallah (20I0, p. 59I), os desafios no setor de saúde se ampliaram bastante nos últimos anos, devido a fatores como o "aumento contínuo dos custos com a saúde, a expansão das demandas do mercado, o surgimento de novas doenças e tratamentos, os avanços tecnológicos, a conscientização pública, a crescente insatisfação do cliente e a atenção da mídia”. 
Em um esforço para cortar custos e, assim, permanecer competitivas, as instituições de saúde vêm tomando sérias medidas para melhorar o desempenho (WICKRAMASINGHE, 2008). Nesse contexto, e devido à alta competitividade, as empresas do setor se veem forçadas a adotar estratégias que garantam a sustentabilidade dos negócios e mitiguem os conflitos de interesses, de forma condizente com os preceitos da Teoria da Legitimidade, segundo os quais as empresas buscam mecanismos para atender às expectativas da sociedade.

A Teoria da Legitimidade tem como pressuposto que o gestor adotará estratégias no sentido de demonstrar para os stakeholders que a empresa procura atender a suas expectativas (CHAN; WATSON; WOODLIFF, 20I4). Desse modo, para se legitimar no mercado, a empresa precisa modificar a percepção dos stakeholders quanto às suas ações (SUCHMAN, I995), de maneira que se apresentem de forma desejável, apropriadas ou adequadas, pois é por meio da legitimidade que a empresa se perpetua (DOWLING; PFEFFER, I975).

Dentre as diversas práticas voltadas para garantir a sustentabilidade nos negócios e mitigar os conflitos de interesses, e assim atender às diversas expectativas dos stakeholders, destaca-se a sinalização ao mercado, por meio do disclosure, da adoção de melhores práticas de governança corporativa e de responsabilidade social corporativa, o que culmina com a melhoria do desempenho empresarial (BENNINGTON, 20I0; MONFARDINI; BARRETTA; RUGGIERO, 20I3).

A governança corporativa coordena a eficiência do relacionamento entre a empresa e acionistas, gestores e colaboradores (CADBURY, I993), e compreende um conjunto de mecanismos (HART, I995) capazes de garantir a rentabilidade dos investimentos realizados pelos acionistas (SHLEIFER; VISHNY, 1997). A responsabilidade social corporativa, por sua vez, tem foco nos compromissos e deveres (sociais e ambientais) perante seus stakeholders, além da criação e captura de valor para os acionistas (AGUILERA et al., 2007), caracterizando-se no triple bottom line, em que a empresa cria estratégias de desenvolvimento sustentável baseado nos aspectos econômico, ambiental e social (BROOKS; OIKONOMOU, 2018).

Tanto a responsabilidade social corporativa como a governança corporativa impactam o desempenho empresarial (DEL BOSCO; MISANI, 20I6; BROOKS; OIKONOMOU, 20I8; JO; HARJOTO, 20I I; NTIM; SOOBAROYEN, 20I3), e contribuem para viabilizar a legitimidade da empresa. Apesar disso, poucos estudiosos procuraram entender o alinhamento entre ambos (MONEY; SCHEPERS, 2007) e como esses fatores impactam o valor e o desempenho da empresa (ABOUD; DIAB, 20I8; ECCLES; IOANNOU; SERAFEIM, 20I4; FATEMI; GLAUM; KAISER, 20I7; LI et al., 2018), contribuindo para a perpetuidade da empresa no mercado.

Diante do exposto, e considerando a relevância desses fatores para o setor de saúde, o presente estudo tem por objetivo identificar o efeito da responsabilidade social corporativa e da governança corporativa no desempenho empresarial no setor de saúde.

Para tanto, e por meio de regressão quantílica com dados em painel desbalanceado, foram analisados os dados de 453 empresas de capital aberto de 3I países, referentes ao período de 2010 a 2018, totalizando I.825 observações. Foram utilizados os indicadores Environmental, Social and Governance (ESG), da Thomson Reuters Eikon, que mensuram as práticas de responsabilidade ambiental e social e a governança corporativa. Destaque-se que o índice criado por meio dos indicadores ESG constitui uma proxy abrangente, capaz de captar a responsabilidade ambiental e social e a governança corporativa. O desempenho empresarial, por sua vez, é mensurado pelo Return On Assets (ROA) e pelo Q de Tobin adaptado, conforme utilizado por Buallay (2019).

Com base na revisão de literatura, e considerando-se os preceitos da Teoria da Legitimidade, conjectura-se que quanto mais elevado for o nível de adoção de melhores práticas de governança e de responsabilidade social corporativa, maior será o desempenho empresarial.

Destaque-se a relevância deste estudo, pois, segundo Geiger, Marlin e Segrest (2019), no setor de saúde o ambiente é complexo e incerto, além de impactar o bem-estar humano e representar uma parcela considerável do Produto Interno Bruto (BENNINGTON, 20I0), exigindo grande esforço da empresa para equilibrar eficiência, qualidade e segurança na tentativa de garantir sua continuidade. Apesar disso, o segmento ainda é pouco investigado na literatura nacional e estrangeira, carecendo de 
discussão especialmente no que diz respeito à correlação entre desempenho empresarial, governança corporativa e responsabilidade social corporativa (EECKLOO et al., 2004; JAMALI; HALLAL; ABDALLAH, 20I0; POINTER; ORLIKOFF, I999), levando-se em conta uma análise cross-country.

Destarte, além de contribuir com a literatura ao estender o conhecimento sobre o relacionamento entre as práticas de responsabilidade social corporativa e a governança corporativa das empresas do setor de saúde e sinalizar o impacto de variáveis macroeconômicas, tais como o Produto Interno Bruto e o Índice de Desenvolvimento Humano, no desempenho empresarial, o estudo adiciona insights sobre como a responsabilidade social corporativa (ambiental e social) e a governança corporativa, conjuntamente, afetam o desempenho operacional e de mercado das companhias do setor de saúde de vários países.

\section{REVISÃO DA LITERATURA}

Embora a origem da governança e da responsabilidade social tenha merecido abordagens distintas, as preocupações e os problemas com essas estratégias corporativas convergem para questões relacionadas a ética, prestação de contas e divulgação (LERACH, 2002), principalmente devido às pressões de desempenho e às crescentes expectativas de partes interessadas.

Além de adotar regras e mecanismos para monitorar o comportamento de gestores e reduzir os conflitos de agência com os investidores (governança corporativa), a empresa também procura equilibrar as necessidades de grupos díspares com as metas dos acionistas, nos campos social e ambiental (responsabilidade social corporativa) (MCBARNET; VOICULESCU; CAMPBELL, 2007). Para tanto, importa estabelecer um diálogo com as partes interessadas e procurar conquistar o reconhecimento da sociedade.

Dessa forma, e sob os preceitos da Teoria da legitimidade, os gestores devem divulgar as estratégias voltadas para o atendimento das expectativas da sociedade (DEEGAN, 2002), buscando o reconhecimento das operações da empresa (CHAN; WATSON; WOODLIFF, 20I4). A Teoria da Legitimidade se baseia no pressuposto de que os gerentes adotarão estratégias para demonstrar que a organização vem tentando cumprir seus contratos (implícitos e explícitos) com a sociedade.

Holder-Webb et al. (2009) argumentam que somente aquilo que é divulgado pode provocar mudanças na percepção dos stakeholders. Considerando-se as temáticas deste estudo, Elliott et al. (20I4) relatam que os efeitos da divulgação de responsabilidade social corporativa podem depender da qualidade da governança, sugerindo que a confiabilidade e o conteúdo das informações são pesos importantes para os investidores (CORAM; MONROE; WOODLIFF, 2009) e, portanto, para a legitimidade da empresa.

A literatura pertinente tem revelado que as estratégias de governança corporativa são fortemente correlacionadas com as de responsabilidade social corporativa (BELTRATTI, 2005; KAYMAK; BEKTAS, 20I7; MICHELON; PARBONETTI, 20I2). A governança corporativa estabelece mecanismos de controle para mitigar conflitos de interesses entre agentes (gestores) e principal (acionistas), definindo, por exemplo, como a empresa funciona em sua formação, estruturação, deveres, relacionamentos e responsabilidade. Dentre os aspectos de governança corporativa, destacam-se aqueles referentes à composição do conselho de administração, tais como a separação dos cargos do presidente e do CEO e a presença significativa de diretores externos e independentes (CHEN; LI; SHAPIRO, 20II).

Em atenção aos interesses das diversas partes envolvidas, a governança corporativa evoluiu de uma compreensão econômica e legalista para uma busca de equilíbrio entre os diferentes stakeholders (MASON; SIMMONS, 20I4; REINHARDT; STAVINS; VIETOR, 2008). Nesse sentido, a governança relaciona-se à responsabilidade social corporativa, que também tem foco no compromisso contínuo de um negócio que se comporta de forma ética em relação à sociedade maior e a seus acionistas. 
A responsabilidade social corporativa estabelece estratégias fundamentadas nos aspectos econômico, social e ambiental, caracterizados como triple bottom line (BROOKS; OIKONOMOU, 2018), e busca o alinhamento das diversas partes interessadas. Trata-se de uma estratégia que conecta sistemicamente a empresa, suas práticas e responsabilidades ao seu valor e desempenho (BARNEA; HEINKEL; KRAUS, 2013) e aos interesses da sociedade, por meio de compromissos corporativos, estratégia, operações, relações constituintes e governança (PORTER; KRAMER, 20II).

Dentre os principais elementos da responsabilidade social corporativa, destacam-se práticas éticas, transparência, meio ambiente, saúde e segurança, integridade de produto e marketing, envolvimento dos colaboradores, respeito à comunidade, responsabilidade da cadeia de fornecimento e antissuborno e anticorrupção (ANDRIOF; WADDOCK, 2002), todos condizentes com os princípios da governança corporativa (TRICKER, 2009).

Alguns estudiosos concluíram que a empresa com estrutura de governança mais forte tem maior probabilidade de adotar atividades de responsabilidade social corporativa, e que provavelmente também desfrutam de um maior valor no mercado (BROOKS; OIKONOMOU, 20I8; BUALLAY, 2019; JO; HARJOTO 20II; NTIM; SOOBAROYEN, 20I3). A relevância dos indicadores de desempenho da responsabilidade social corporativa para os participantes do mercado depende, geralmente, da credibilidade nos processos empresariais e das práticas de governança corporativa adotadas (HART; MILSTEIN, 2003; TRICKER, 2009), e resultam em desempenho superior e criação de valor (BARNEA; HEINKEL; KRAUS, 20I3; ECCLES; IOANNOU; SERAFEIM, 20I4; FATEMI; GLAUM; KAISER, 20I7; PEIRIS; EVANS, 20I0).

Entretanto, a literatura acadêmica também apresenta pesquisas que relatam uma associação não significativa entre os indicadores de governança e os de responsabilidade social corporativa e o desempenho econômico-financeiro ou valor da empresa (McWILLIAMS; SIEGEL, 2000; PLUMLEE et al., 2015). Esses resultados são geralmente justificados com base na Teoria Neoclássica, defendida por Friedman (1970), em que a maximização do lucro é a única responsabilidade social da empresa.

Apesar disso, segundo a literatura fundamentada na conformidade e nas perspectivas criativas que aumentam a avaliação de riscos e de oportunidades, a responsabilidade social e a governança corporativa contribuem para a criação de valor da empresa, por meio de fatores como a inovação (HART, 20I0), a reputação (FOMBRUN; SHANLEY, I990; IOANNOU; SERAFEIM, 20I2), o melhor relacionamento com stakeholders (BURKE, 2005) e a gestão de riscos (STEINGRABER; KANE, 2010). Eccles, Ioannou e Serafeim (20I4) afirmam que a empresa que adota políticas de responsabilidade social supera suas contrapartes no mercado de ações. De forma similar, Jo e Harjoto (20II) observam que a empresa que também adota melhores práticas de governança corporativa apresenta desempenho superior.

A maioria das pesquisas sobre governança corporativa e responsabilidade social corporativa é aplicada às empresas indistintamente. Setores específicos como o de saúde - objeto deste estudo - têm sido preteridos, ainda que constitua um segmento complexo e que exige elevado nível de qualidade e segurança da organização, a fim de gerar vantagem competitiva. Jamali, Hallal e Abdallah (2010) afirmam que são amplos os desafios enfrentados pela organização de serviços de saúde, destacando-se o aumento dos custos, o menor nível de investimento no setor e a escassez de mão de obra qualificada, e ainda persistindo as preocupações com relação à transparência e à responsabilidade da empresa.

Embora o setor de saúde não seja o centro das atenções no campo das iniciativas de responsabilidade social corporativa, é considerado relevante tanto no campo econômico como no social (JAMALI; HALLAL; ABDALLAH, 20I0). No contexto da governança, Chambers (2012) destaca a importância dos conselhos de administração das firmas de saúde na medida em que assumem a responsabilidade final pela qualidade dos produtos e serviços ofertados à sociedade.

No setor de saúde são raros os estudos sobre governança e responsabilidade social (BENNINGTON, 20I0; JAMALI; HALLAL; ABDALLAH, 20I0; MONFARDINI; BARRETTA; RUGGIERO, 20I3), e não foram identificadas pesquisas que relacionam tais temas com as características das empresas e dos seus países-sede. Assim, o presente estudo considera os aspectos do desenvolvimento econômico e social, regulamentações legais do país em que a empresa atua, conforme 
pesquisa realizada com empresas de distintos setores econômicos (CUERVO, 2002; LA PORTA et al., I998; REAZ; HOSSAIN, 2007).

Diante do exposto, e com base na revisão de literatura, espera-se uma relação positiva entre a governança corporativa e a responsabilidade social corporativa, a partir do que se formula a primeira hipótese da pesquisa:

Hipótese I: Há uma relação positiva entre a responsabilidade social corporativa e a governança corporativa.

Assim como se supõe que quanto maior for o nível de adoção de melhores práticas de governança e de responsabilidade social corporativa, maior será o desempenho da empresa do setor de saúde, levando-se em conta suas características e a de seus países-sede. Tem-se, portanto, as seguintes hipóteses de pesquisa:

Hipótese 2: Quanto maiores forem a responsabilidade social corporativa e a governança corporativa, maior será o desempenho operacional.

Hipótese 3: Quanto maiores forem a responsabilidade social corporativa e a governança corporativa, maior será o desempenho de mercado.

\section{METODOLOGIA}

Trata-se de estudo teórico-empírico, que pode ser caracterizado quanto à abordagem, objetivos e procedimentos. Quanto à abordagem, consiste em pesquisa quantitativa, por empregar técnicas estatísticas. Quanto aos objetivos, é uma pesquisa descritiva, por descrever a correlação entre governança corporativa, responsabilidade social corporativa e desempenho empresarial. E quanto aos procedimentos é uma pesquisa documental, por utilizar as bases de dados da Thomson Reuther Eikon e do World Bank.

A população da pesquisa compreende todas as empresas do setor de saúde listadas em mercados de capitais. Para a composição da amostra foram definidos alguns requisitos: (i) os dados das empresas sobre governança e responsabilidade social corporativa devem estar disponíveis na base da Thomson Reuters Eikon, em pelo menos um dos exercícios entre 2010 e 2018; e (ii) as empresas devem disponibilizar dados econômico-financeiros para a mensuração do desempenho empresarial e das variáveis de controle. Desse modo, a amostra final reuniu 453 empresas de 3 I países, totalizando I.825 observações, distribuídas conforme mostra a Tabela I.

Tabela I - Distribuição das I.825 observações da amostra por país - 20I0-2018

\begin{tabular}{llll}
\hline País & Número de observações & País & Número de observações \\
\hline África do Sul & 29 & Indonésia & 7 \\
Alemanha & $7 \mathrm{I}$ & Irlanda & 45 \\
Austrália & $\mathrm{I} 47$ & Israel & I I \\
Bélgica & 22 & Itália & 2 \\
Brasil & $\mathrm{I}$ & Japão & $\mathrm{I} 83$ \\
Canadá & 40 & Coreia do Sul & $\mathrm{I} 8$ \\
China & $5 \mathrm{I}$ & Luxemburgo & 3 \\
Cingapura & $\mathrm{I} 7$ & Malásia & 8 \\
Dinamarca & 49 & México & 3 \\
Espanha & $\mathrm{I} 8$ & Nova Zelândia & $\mathrm{I} 8$ \\
Estados Unidos & 775 & Países Baixos & 18 \\
Finlândia & $\mathrm{I} 6$ & Reino Unido & 82 \\
França & 27 & Suécia & 20 \\
Hong Kong & 8 & Suíça & 64 \\
Hungria & 7 & Tailândia & 8 \\
Índia & 57 & & \\
\hline
\end{tabular}


A delimitação da pesquisa entre 2010 e 2018 deve-se ao fato de esse período ser marcado pelos efeitos da crise financeira mundial que teve início em 2007 e finalizou em 2009 (Scott, 2015).

Para análise de rejeição ou não de cada hipótese de estudo, foram aplicadas técnicas estatísticas. Para análise da Hipótese I foi empregada a correlação de Pearson, a fim de se observar se a responsabilidade social corporativa e a governança corporativa são positivamente correlacionadas.

Para análise das Hipótese 2 e 3, foi aplicada a regressão quantílica com dados em painel desbalanceado, erros-padrão robustos e efeitos fixos de ano. Adotou-se a regressão quantílica, pois, nesse método, a estimativa dos parâmetros funciona de forma não paramétrica $\mathrm{e}$, considerando que utiliza a mediana em vez da média, não requer o atendimento aos pressupostos do método dos mínimos quadrados ordinários (OLS), evitando a estimação de parâmetros ineficientes e erros-padrão viesados, resultantes de problemas de heterogeneidade e da presença de outliers (FÁVERO; BELFIORE, 20I7; YU; WANG; WU, 20I5).

Assim, com o objetivo de se verificar o efeito da responsabilidade social corporativa e da governança corporativa no desempenho empresarial, foi utilizado o modelo representado na Equação I.

$$
\begin{aligned}
D E S_{i t}=\beta_{0}+ & \beta_{1} E S G_{i t}+\beta_{2} T_{A M M_{i t}}+\beta_{3} C R E S_{i t}+\beta_{4} E N D_{i t}+\beta_{5} F C O_{i t}+\beta_{6} P I B_{i t} \\
& +\beta_{7} I D H_{i t}+\beta_{8} I G N_{i t}+\text { Ano }+\varepsilon_{i t}
\end{aligned}
$$

Destaca-se que o desempenho empresarial (DES) comporta duas abordagens: operacional e de mercado (BUALLAY, 2019). O desempenho empresarial operacional adota como proxy o Retorno sobre o Ativo (ROA), dado pela razão entre o lucro antes de itens extraordinários e o Ativo. O desempenho empresarial de mercado utiliza como proxy o Q de Tobin adaptado (QTOB), mensurado mediante divisão da soma do valor de mercado com a dívida total pelo Patrimônio Líquido.

A responsabilidade social corporativa e a governança corporativa são mensuradas pelos pilares do índice ESG, da Thomson Reuters Eikon (ABOUD; DIAB, 20I8; BUALLAY, 20I9).

A responsabilidade social corporativa (RSC) é mensurada pelos pilares ambiental (AMB), composto por três dimensões (Resource Use, Emissions e Innovation); e social (SOC), composto por quatro dimensões (Workforce, Human Rights, Community e Product Responsibility). As dimensões do pilar ambiental (AMB) incluem 6I assertivas (Resource Use $=19 ;$ Emissions $=22$; Innovation $=$ 20), enquanto as dimensões do pilar social (SOC) incluem 63 assertivas (Workforce $=29$; Human Rights = 8; Community = I4; e Product Responsibility = I2). Quanto maior for RSC, maior é a responsabilidade social corporativa.

A governança corporativa (GOV) é mensurada pelo pilar composto pelas dimensões Management, Shareholders e CSR Strategy, com 34, I2 e 8 assertivas, respectivamente. Quanto maior for GOV, maior é a governança corporativa.

Para se verificar o efeito conjunto da responsabilidade social corporativa e da governança corporativa, foi utilizado o escore ESG, definido pela Thomson Reuters Eikon. Desse modo, quanto maior for o escore ESG, maiores serão a responsabilidade social corporativa e a governança corporativa.

No presente estudo foram considerados dois conjuntos de variáveis de controle, tendo em vista os contextos empresa e país, sendo obtidas na Thomson Reuters Eikon e no World Bank, respectivamente. No contexto empresa, as variáveis são: (i) o tamanho da empresa (TAM), mensurado pelo logaritmo natural do ativo (ABOUD; DIAB, 2018; BUALLAY, 2019; XIE et al., 2019); (ii) o crescimento da receita (CRES), mensurado pela variação proporcional da receita líquida (LONIAL et al., 2008; WANG; SARKIS, 20I7); (iii) o endividamento (END), calculado mediante divisão da dívida total pelo ativo (ABOUD; DIAB, 20I8; WANG; SARKIS, 2017); e (iv) a geração de caixa (FCO), mensurada pela divisão do fluxo de caixa operacional pelo ativo (GARCIA; MENDES-DASILVA; ORSATO, 20I7; LONIAL et al., 2008).

No contexto país, as variáveis são: (i) o Produto Interno Bruto (PIB), dado pelo logaritmo natural do PIB nacional (CRIFO; DIAYE; OUEGHLISSI, 20I7; MARGARETIC; POUGET, 20I8); (ii) o Índice de Desenvolvimento Humano (IDH) (CRIFO; DIAYE; OUEGHLISSI, 20I7; 
MARGARETIC; POUGET, 20I8); e (iii) o Índice de Governança Nacional (IGN), mensurado por meio da análise fatorial dos indicadores Voz e Responsabilização, Estabilidade Política, Eficácia do Governo, Qualidade Regulatória, Estado de Direito e Controle da Corrupção, propostos por Kaufmann, Kraay e Zoido-Lobatón (I999) (CRIFO; DIAYE; OUEGHLISSI, 2017; MARGARETIC; POUGET, 20I8). Frise-se que as variáveis passaram pelo processo de winsorização ao nível de I\% nos extremos, para correção de outliers.

\section{ANÁLISE E DISCUSSÃO DOS RESULTADOS}

As empresas do setor de saúde representam uma parcela significativa da economia nacional, e desempenham uma função social relevante, razão pela qual são sensíveis às variações do mercado e da economia. Nesse sentido, com base nos dados da pesquisa, a Figura I mostra a evolução do desempenho empresarial operacional e de mercado (ROA e Q de Tobin) e do escore ESG das empresas da amostra, assim como do Produto Interno Bruto (PIB), do Índice de Desenvolvimento Humano (IDH) e do Índice de Governança Nacional (IGN) dos respectivos países-sede.

Figura I - Evolução dos indicadores

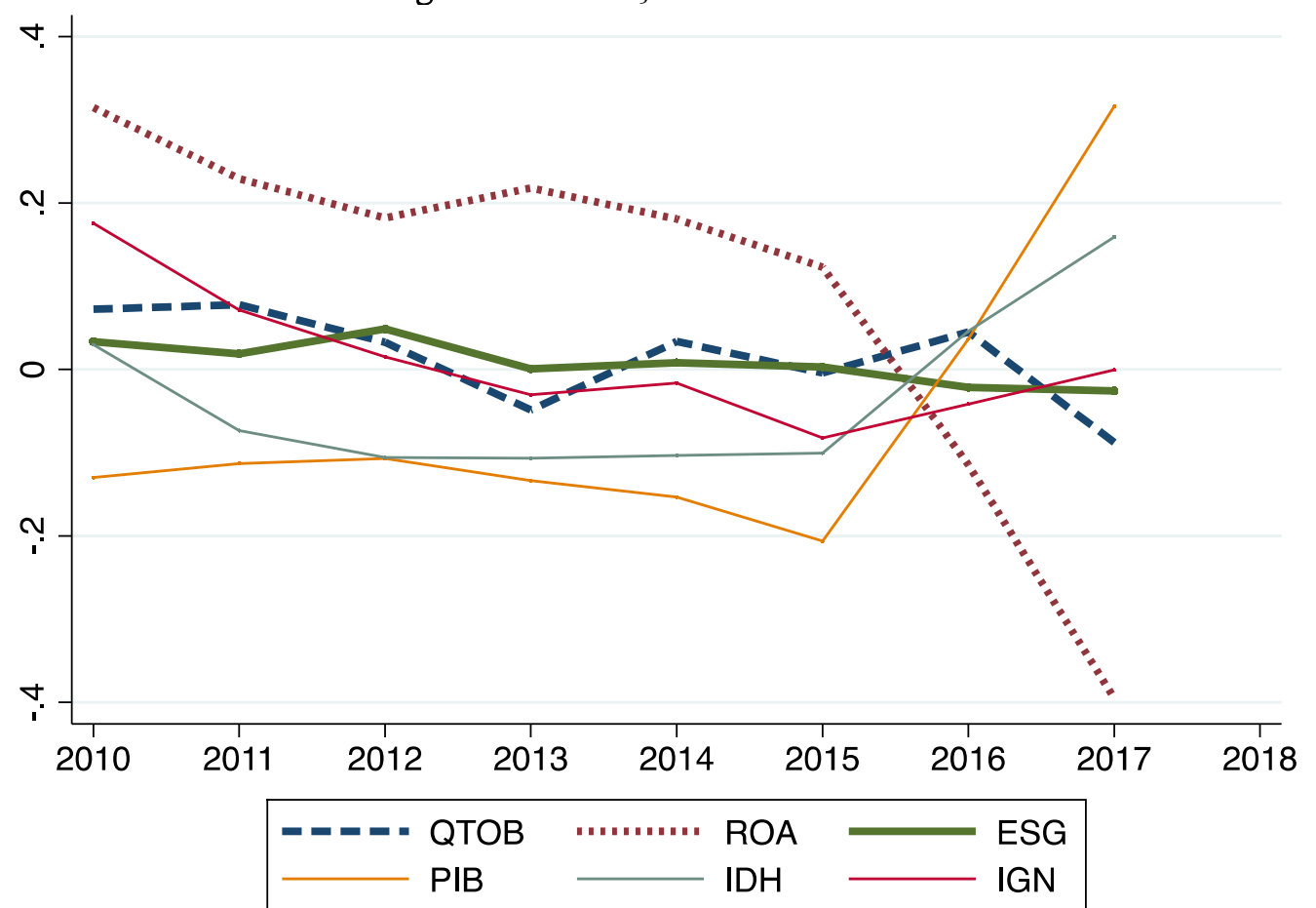

Nota. $\mathrm{QTOB}=\mathrm{Q}$ de Tobin; $\mathrm{ROA}=$ Retorno sobre o Ativo; $\mathrm{ESG}=$ escore ESG; PIB = Produto Interno Bruto; IDH = Índice de Desenvolvimento Humano; IGN = Índice de Governança Nacional. Para fins de comparação, os valores das variáveis foram padronizados.

Ao se observar o comportamento das variáveis ao longo do tempo, percebe-se que o ano 2015 impactou as empresas e os países, especialmente quanto ao desempenho operacional (ROA), ao Produto Interno Bruto (PIB) e ao Índice de Desenvolvimento Humano (IDH). Enquanto isso, o ROA (Teste F $=\mathrm{I0}, 2 \mathrm{I} ; p$-value $=0,000)$ das empresas do setor de saúde diminui bruscamente, e o PIB (Teste $\mathrm{F}=$ I8,44; $p$-value $=0,000)$ e o IDH (Teste $\mathrm{F}=3,06 ;$ p-value $=0,003)$ dos países cresceram; esse resultado foi confirmado por meio da análise de variância. Essa queda no desempenho das empresas, especialmente em 20I5, pode ser justificada pela crise no mercado acionário, impulsionada pela instabilidade do mercado dos EUA, pela desaceleração da economia chinesa e pela crise de dívida de países europeus, como a Grécia, por exemplo (TIME MAGAZINE, 20I5). 
Quanto ao escore ESG, percebe-se que, apesar de se verificar uma pequena redução, as práticas de responsabilidade social corporativa e governança corporativa foram constantes (Teste $\mathrm{F}=0,15$; $p$ value $=0,9936)$, indicando um certo conservadorismo das empresas da amostra quanto a essas estratégias de legitimação. Por sua vez, tanto o $Q$ de Tobin (Teste $F=0,95$; $p$-value $=0,4653$ ) quanto o IGN (Teste F = I,09; $p$-value $=0,365 \mathrm{I}$ ) oscilaram durante o período de análise, demonstrando uma queda em ambos os indicadores, apesar de não haver diferença significativa entre os anos.

Desse modo, como o desempenho empresarial, em particular o desempenho operacional, diminuiu ao longo do tempo e não houve variação nos indicadores das práticas de responsabilidade social corporativa, pode-se, assim, esperar uma relação inversa.

Para se compreender melhor o comportamento de cada variável de interesse e complementar à análise realizada na Figura I, foi aplicada a estatística descritiva, conforme explicitado na Tabela 2.

Tabela 2 - Estatística descritiva

\begin{tabular}{|c|c|c|c|c|c|}
\hline Variável & Número de Observações & Média & Desvio-padrão & Mínimo & Máximo \\
\hline ROA & I 825 & 0,019 & 0,166 & $-0,929$ & 0,305 \\
\hline QTOB & 1825 & 7,952 & $\mathrm{I} 2,324$ & $-34,744$ & 150,613 \\
\hline ESG & 1825 & 0,507 & 0,172 & 0,127 & 0,879 \\
\hline AMB & I 825 & 0,499 & 0,206 & 0,087 & 0,955 \\
\hline SOC & I 825 & $0,5 \mathrm{I} 2$ & 0,220 & $0,08 \mathrm{I}$ & 0,945 \\
\hline GOV & 1825 & $0,5 \mathrm{II}$ & $0,2 \mathrm{II}$ & 0,077 & 0,930 \\
\hline TAM & I 825 & $2 \mathrm{I}, 764$ & $\mathrm{I}, 792$ & 16,468 & 25,996 \\
\hline CRES & 1825 & 0,229 & 0,828 & $-0,926$ & 6,169 \\
\hline END & 1825 & 0,220 & 0,190 & 0,000 & 0,948 \\
\hline FCO & 1825 & $0,06 \mathrm{I}$ & $0, \mathrm{I} 46$ & $-0,65 \mathrm{I}$ & 0,374 \\
\hline PIB & 1825 & 29,080 & 1,499 & 24,770 & 30,601 \\
\hline $\mathrm{IDH}$ & 1825 & 0,898 & 0,069 & $0,58 \mathrm{I}$ & 0,944 \\
\hline IGN & 1825 & 0,278 & 0,768 & $-2,427$ & I, 282 \\
\hline
\end{tabular}

Nota. $\mathrm{ROA}=$ Retorno sobre o Ativo; QTOB = Q de Tobin; ESG = Escore ESG; AMB = Pilar ambiental do ESG; SOC = Pilar social do ESG; GOV = Pilar governança do ESG; TAM = Tamanho da empresa; CRES = Crescimento da receita END = Endividamento; FCO = Geração de caixa; PIB = Produto Interno Bruto; IDH = Índice de Desenvolvimento Humano; IGN = Índice de Governança Nacional.

Apesar de na Figura I o ROA e o $Q$ de Tobin apresentarem uma diminuição ao longo do período de análise, a média de ambas as variáveis é positiva. Quanto às variáveis que representam as práticas de responsabilidade social corporativa (AMB e SOC) e de governança corporativa (GOV), nota-se que as empresas da amostra possuem um valor mediano, já que equivalem a aproximadamente $50 \%$ de uma escala de 0 a 100 .

Considerando-se que ao longo do período as empresas do setor de saúde não variam as suas estratégias quanto às práticas responsabilidade social corporativa e de governança corporativa, como demonstrado na Figura I, e que tais práticas são medianas, elas teriam maior dificuldade para se legitimar no mercado, e, assim, auferir melhor desempenho empresarial, além de reduzir conflitos de interesses e atender às expectativas dos stakeholders.

Quanto às características das empresas do setor de saúde, considerando a variável tamanho (TAM), observa-se que todas são grandes, encontram-se em crescimento, possuem baixo endividamento e vêm gerando caixa, conforme observado nas variáveis endividamento (END) e geração de caixa (FCO), favorecendo o alcance de um melhor desempenho empresarial. Quanto aos atributos dos países, percebe-se que a maioria das empresas da amostra encontra-se instalada em países de alto desenvolvimento socioeconômico, sendo $42 \%$ nos Estados Unidos, que apresentam indicadores compatíveis com a potencial geração de benefícios econômicos para essas empresas, já que são menos suscetíveis a adversidades. 
Para analisar a relação entre as variáveis do estudo e testar a Hipótese I, de que há uma relação positiva entre a responsabilidade social corporativa (AMB e SOC) e a governança corporativa (GOV), foi realizada a correlação de Pearson (Tabela 3 ).

Tabela 3 - Correlação de Pearson

\begin{tabular}{|c|c|c|c|c|c|c|c|c|c|c|c|c|c|}
\hline & $\mathrm{ROA}$ & QTOB & ESG & $\mathrm{AMB}$ & SOC & GOV & TAM & CRES & END & $\mathrm{FCO}$ & PIB & IGN & $\mathrm{IDH}$ \\
\hline $\mathrm{ROA}$ & I & & & & & & & & & & & & \\
\hline QTOB & $-0,034$ & I & & & & & & & & & & & \\
\hline ESG & $\left.0,209^{*}\right)$ & $-0,048^{(\dagger)}$ & I & & & & & & & & & & \\
\hline $\mathrm{AMB}$ & $0,2 \mathrm{I} 5^{(*)}$ & $-0,039$ & $0,84 \mathrm{I}^{(*)}$ & I & & & & & & & & & \\
\hline SOC & $0,159^{(*)}$ & $\left.-0,062^{\circ}\right)$ & $\left.0,877^{\circ}\right)$ & $\left.0,67 \mathrm{I}^{\circ}\right)$ & I & & & & & & & & \\
\hline GOV & $\left.0,130^{*}\right)$ & $-0,0$ I I & $\left.0,696^{\circ}\right)$ & $\left.0,348^{\circ}\right)$ & $\left.0,400^{\circ}\right)$ & I & & & & & & & \\
\hline TAM & $0,397^{(*)}$ & $\left.-0,15 I^{*}\right)$ & $0,575^{(*)}$ & $\left.0,496^{\circ}\right)$ & $0,5 \mathrm{I} 7^{(*)}$ & $0,372^{(*)}$ & I & & & & & & \\
\hline CRES & $-0,173^{(*)}$ & $0,06 \mathrm{I}^{(*)}$ & $-0, \mathrm{I} I 6^{(*)}$ & $-0, I 30^{*}$ & $\left.-0,069^{*}\right)$ & $-0,086^{*}$ & $-0,162^{(*)}$ & I & & & & & \\
\hline END & $-0,007$ & $0,053^{(\dagger)}$ & $0, \mathrm{I} 44^{*}$ & $0,108^{(*)}$ & $0,184^{(*)}$ & $0,044^{(\ddagger)}$ & $0,255^{\circ}$ & $-0,054^{(\dagger)}$ & I & & & & \\
\hline FCO & $\left.0,885^{*}\right)$ & $-0,026$ & $\left.0,233^{*}\right)$ & $0,227^{(*)}$ & $\left.0,185^{\circ}\right)$ & $\left.0,149^{\circ}\right)$ & $0,4 \mathrm{II}^{(*)}$ & $-0,207^{(*)}$ & $0,039^{\ddagger)}$ & I & & & \\
\hline PIB & $\left.-0,090^{*}\right)$ & $0,02 \mathrm{I}$ & $-0,016$ & $-0,0 \mathrm{II}$ & $-0,043^{(\ddagger)}$ & 0,018 & $0,090^{*}$ & $0,058^{(\dagger)}$ & $0,098^{(*)}$ & $-0,064^{(*)}$ & I & & \\
\hline IDH & $-0,17 I^{(*)}$ & $0,074^{* *}$ & $0,074^{(*)}$ & $0,086^{(*)}$ & $0, \mathrm{II} 5^{(*)}$ & $-0,032$ & 0,007 & $-0,0 \mathrm{I3}$ & $0,02 \mathrm{I}$ & $-0,095^{(*)}$ & $0,162^{*}$ & I & \\
\hline IGN & $\left.-0,134^{\circ}\right)$ & $0,08 \mathrm{I}^{(*)}$ & $0,124^{(*)}$ & $\left.0,116^{\circ}\right)$ & $0,168^{(*)}$ & 0,005 & $-0,0 \mathrm{I} 4$ & $-0,019$ & $0,0 \mathrm{II}$ & $-0,072^{\circ}$ & $-0,108^{(*)}$ & $\left.0,897^{*}\right)$ & $\mathrm{I}$ \\
\hline
\end{tabular}

Nota. $\left(^{*}\right)$ Significância ao nível de 0,0I; $\left(^{+}\right)$significância ao nível de 0,05; $\left(^{(}\right)$significância ao nível de 0,I.

$\mathrm{ROA}=$ Retorno sobre o Ativo; $\mathrm{QTOB}=\mathrm{Q}$ de Tobin; ESG = Escore ESG; AMB = Pilar ambiental do ESG; SOC = Pilar social do ESG; GOV = Pilar governança do ESG; TAM = Tamanho da empresa; $C R E S$ = Crescimento da receita; END = Endividamento; FCO = Geração de caixa; PIB = Produto Interno Bruto; IDH = Índice de Desenvolvimento Humano; IGN = Índice de Governança Nacional.

De modo condizente com a literatura pertinente, a Hipótese I não é rejeitada, porque as práticas de responsabilidade social corporativa ( $\mathrm{AMB}$ e $\mathrm{SOC}$ ) são positivamente correlacionadas com as práticas de governança corporativa (GOV), de maneira que, quanto maior for uma, maior será a outra, simultaneamente (BELTRATTI, 2005; KAYMAK; BEKTAS, 20I7; MICHELON; PARBONETTI, 2012).

Quanto ao desempenho, as abordagens operacional (ROA) e de mercado (QTOB) não apresentaram relação significativa, divergindo de estudos que demonstram uma relação positiva e significante entre o desempenho operacional e o de mercado (ABOUD; DIAB, 20I8; BUALLAY, 2019; YU; GUO; LUU, 2018). Contudo, como o desempenho de mercado inclui expectativas dos stakeholders, tidas como intangibilidade da empresa e menos sensibilidade ao gerenciamento de resultados, isso pode gerar divergência de resultados (LIOUI; SHARMA, 20I2).

Analisando-se as relações entre a responsabilidade social corporativa (AMB e SOC), a governança corporativa (GOV) e o desempenho empresarial (ROA e QTOB), percebe-se que há uma relação significante e inversa entre $\mathrm{AMB}$, SOC e GOV com o QTOB e uma relação positiva e significante entre $\mathrm{AMB}, \mathrm{SOC}$ e GOV com o ROA. Esse resultado é condizente com a literatura pertinente (WALLS; BERRONE; PHAN, 20I2), já que os achados ainda são conflitantes, mesmo não se considerando nenhuma variável de controle, o que poderia sugerir a rejeição da Hipótese 3 para a abordagem de mercado (QTOB) do desempenho empresarial.

As variáveis de país, especialmente o IDH e o IGN, têm relação significante com o desempenho empresarial (ROA e QTOB), a responsabilidade social corporativa e as práticas conjuntas de responsabilidade social corporativa e da governança corporativa (ESG). Quanto maior for o desenvolvimento social (IDH e IGN) do país, maiores serão o desempenho de mercado (QTOB), as práticas de responsabilidade social corporativa (AMB e SOC) e as práticas conjuntas de 
responsabilidade social corporativa e governança corporativa (ESG). Ao mesmo tempo, o desenvolvimento social (IDH e IGN) tem uma relação negativa com o desempenho operacional (ROA).

Percebe-se, portanto, que o ambiente institucional é relevante para que as empresas adotem práticas de legitimação que geram expectativas perante os stakeholders, refletindo-se no desempenho de mercado.

Como a correlação de Pearson desconsidera outros fatores na relação entre as variáveis, foi realizada a regressão quantílica com erros-padrão robustos e efeitos fixos de ano, para análise de viabilidade de rejeição ou não das Hipóteses 2 e 3. Para se compreender melhor o efeito das práticas de responsabilidade social corporativa e governança corporativa, a Tabela 4 apresenta, para cada uma das duas abordagens de desempenho empresarial (operacional - ROA e de mercado - QTOB), uma regressão de cada pilar da ESG (ambiental, social e governança), assim como uma regressão do escore ESG que representa a interseção da responsabilidade social corporativa com a governança corporativa.

Tabela 4 - Regressão quantílica

\begin{tabular}{|c|c|c|c|c|c|c|c|c|}
\hline \multirow{2}{*}{ Variável } & $\mathrm{ROA}$ & QTOB & $\mathrm{ROA}$ & QTOB & $\mathrm{ROA}$ & QTOB & $\mathrm{ROA}$ & QTOB \\
\hline & $\mathrm{AMB}$ & $\mathrm{AMB}$ & SOC & SOC & GOV & GOV & ESG & ESG \\
\hline \multirow[t]{2}{*}{ ESG } & 0,000 & $2,673^{(*)}$ & $-0,013^{(*)}$ & $3,189^{(*)}$ & $-0,008^{(*)}$ & $\mathrm{I}, 355^{(*)}$ & $-0,0 \mathrm{I} 3^{(*)}$ & $3,899^{(*)}$ \\
\hline & $(0,46 \mathrm{I})$ & $(0,000)$ & $(0,005)$ & $(0,43 \mathrm{I})$ & $(0,004)$ & $(0,002)$ & $(0,006)$ & $(0,001)$ \\
\hline \multirow[t]{2}{*}{ TAM } & $0,002^{(*)}$ & $-0,90 I^{(*)}$ & $0,003^{(*)}$ & $-0,928^{(\dagger)}$ & $0,002^{(*)}$ & $-0,80 I^{(\dagger)}$ & $0,003^{(*)}$ & $-0,963^{(t)}$ \\
\hline & $(0,00 \mathrm{I})$ & $(0,067)$ & $(0,00 \mathrm{I})$ & $(0,068)$ & $(0,00 \mathrm{I})$ & $(0,062)$ & $(0,00 \mathrm{I})$ & $(0,067)$ \\
\hline \multirow[t]{2}{*}{ CRES } & $-0,002$ & $0,5 \mathrm{II}$ & $-0,003^{(*)}$ & 0,400 & $-0,003(\dagger)$ & 0,427 & $-0,003^{(*)}$ & $0,44 \mathrm{I}$ \\
\hline & $(0,002)$ & $(0,32 \mathrm{I})$ & $(0,00 \mathrm{I})$ & $(0,264)$ & $(0,00 \mathrm{I})$ & $(0,23 \mathrm{I})$ & $(0,002)$ & $(0,276)$ \\
\hline \multirow[t]{2}{*}{ END } & $-0,032^{(*)}$ & 6,670 & $-0,030^{(*)}$ & 5,694 & $-0,032^{(*)}$ & 6,143 & $-0,03 I^{(*)}$ & 6,333 \\
\hline & $(0,005)$ & $(0,538)$ & $(0,005)$ & $(0,545)$ & $(0,004)$ & $(0,563)$ & $(0,004)$ & $(0,5 \mathrm{I} 3)$ \\
\hline \multirow[t]{2}{*}{ FCO } & $0,978^{(*)}$ & $8,670^{(*)}$ & $0,98 \mathrm{I}^{(*)}$ & $8,3 \mathrm{I} 7^{(*)}$ & $0,980^{(*)}$ & $8,98 I^{(\uparrow)}$ & $0,98 \mathrm{I}^{(* *)}$ & $8,983^{(\dagger)}$ \\
\hline & $(0,013)$ & $(0,049)$ & $(0,012)$ & $(0,019)$ & $(0,0 \mathrm{I} 2)$ & $(0,076)$ & $(0,0 \mathrm{I} 2)$ & $(0,037)$ \\
\hline \multirow[t]{2}{*}{ PIB } & $-0,003^{(*)}$ & $0,472^{(t)}$ & $-0,002^{(*)}$ & $0,526^{(\dagger)}$ & $-0,002^{(*)}$ & $0,483^{(t)}$ & $-0,002^{(*)}$ & $0,457^{(\dagger)}$ \\
\hline & $(0,00 \mathrm{I})$ & $(0,052)$ & $(0,00 \mathrm{I})$ & $(0,053)$ & $(0,00 \mathrm{I})$ & $(0,063)$ & $(0,00 \mathrm{I})$ & $(0,056)$ \\
\hline \multirow[t]{2}{*}{$\mathrm{IDH}$} & $-0, \operatorname{II} 8^{(*)}$ & $-2,277$ & $-0,147^{(*)}$ & $-4,820$ & $-0,126^{(+)}$ & $-4,283$ & $-0,136^{(+)}$ & $-2,193$ \\
\hline & $(0,042)$ & $(2,084)$ & $(0,044)$ & $(1,899)$ & $(0,045)$ & $(2,604)$ & $(0,044)$ & $(\mathrm{I}, 607)$ \\
\hline \multirow[t]{2}{*}{ IGN } & $-0,002$ & 0,570 & $0,000^{(*)}$ & 0,735 & $-0,002^{(*)}$ & 0,924 & $-0,00 I^{(*)}$ & 0,616 \\
\hline & $(0,004)$ & $(0,2 \mathrm{I} 2)$ & $(0,004)$ & $(0,183)$ & $(0,004)$ & $(0,263)$ & $(0,004)$ & $(0,147)$ \\
\hline \multirow[t]{2}{*}{ Intercepto } & $0,102^{(\dagger)}$ & 10,172 & $0,107^{(\dagger)}$ & II,713 & $0,095^{(\dagger)}$ & I0,I86 & 0,105 & II 435 \\
\hline & $(0,030)$ & $(\mathrm{I}, 846)$ & $(0,03 \mathrm{I})$ & $(\mathrm{I}, 983)$ & $(0,030)$ & $(2,203)$ & $(0,029)$ & $(\mathrm{I}, 75 \mathrm{I})$ \\
\hline Efeito Fixo - Ano & Sim & Sim & Sim & Sim & Sim & $\operatorname{Sim}$ & Sim & Sim \\
\hline Pseudo $\mathrm{R}^{2}$ & 0,5498 & 0,0564 & 0,5508 & 0,0599 & 0,5502 & 0,0527 & 0,5504 & 0,0589 \\
\hline $\begin{array}{l}N^{o} \text { de Observações } \\
N^{o} \text { de }\end{array}$ & I.825 & 1.825 & I.825 & I.825 & I.825 & I.825 & 1.825 & I. 825 \\
\hline Empresas & 453 & 453 & 453 & 453 & 453 & 453 & 453 & 453 \\
\hline
\end{tabular}

Nota. $\left({ }^{*}\right)$ Significância ao nível de 0,0I; $\left({ }^{+}\right)$significância ao nível de 0,05; ( ${ }^{\ddagger}$ ) significância ao nível de 0,I. Valores entre parênteses representam o erro-padrão robusto.

ROA = Retorno sobre o Ativo; $\mathrm{QTOB}=\mathrm{Q}$ de Tobin; ESG = Escore ESG; AMB = Pilar ambiental do ESG; SOC = Pilar social do ESG; GOV = Pilar governança do ESG; TAM = Tamanho da empresa; CRES = Crescimento da receita; END = Endividamento; FCO = Geração de caixa; PIB = Produto Interno Bruto; IDH = Índice de Desenvolvimento Humano; IGN = Índice de Governança Nacional.

O pilar ambiental (AMB) reflete (i) o desempenho e a capacidade da empresa para reduzir o uso de materiais, energia ou água e de encontrar soluções mais ecoeficientes, melhorando o gerenciamento da cadeia de suprimentos; (ii) o compromisso e a eficácia da empresa no sentido de reduzir as emissões ambientais nos processos operacionais e de produção; e (iii) a capacidade da empresa para reduzir os custos e encargos ambientais para seus clientes, criando novas oportunidades de 
mercado por meio de novas tecnologias e processos ambientais ou produtos ecologicamente projetados (Thomson Reuters, 2019).

$\mathrm{Na}$ Tabela 4, o pilar ambiental (AMB) apresentou resultado significativo apenas no tocante ao desempenho de mercado (QTOB), sendo uma relação positiva, corroborando os achados de Buallay (2019) e Li et al. (2018). As empresas do setor de saúde, portanto, ao destacar suas práticas ambientais, obtêm vantagem competitiva e maior desempenho empresarial, favorecendo a sua legitimação no mercado.

O pilar social (SOC) reflete (i) a eficácia da empresa em relação à satisfação no trabalho e à garantia de um local de trabalho saudável e seguro, mantendo a diversidade e a igualdade de oportunidades e oportunidades de desenvolvimento para sua força de trabalho; (ii) a eficácia da empresa no respeito às convenções fundamentais de direitos humanos; (iii) o compromisso da empresa em ser um bom cidadão, protegendo a saúde pública e respeitando a ética nos negócios; e (iv) a capacidade da empresa para produzir artigos e ofertar serviços de qualidade, integrando a saúde e a segurança do cliente, a integridade e a privacidade dos dados (THOMSON REUTERS, 20I9).

$\mathrm{Na}$ Tabela 4, o pilar social (SOC) apresenta resultado significativo relacionado ao desempenho operacional (ROA) e ao desempenho de mercado (QTOB), sendo, contudo, uma relação negativa e positiva, respectivamente, o que corrobora os achados de Buallay (2019) e Li et al. (2018). Na abordagem do desempenho empresarial, portanto, as empresas do setor de saúde seriam menos lucrativas ao enfatizar essas práticas, podendo os custos e investimentos correspondentes consumir os resultados respectivos. Entretanto, na perspectiva de mercado (desempenho de mercado - QTOB), os stakeholders valorizam tais práticas, o que leva as empresas de saúde a obter vantagem competitiva e maior desempenho, favorecendo a legitimação no mercado.

Considerando-se os pilares ambiental e social, as empresas de saúde que se empenham na responsabilidade social corporativa apresentam uma relação negativa com o desempenho operacional (ROA) e uma relação positiva com o desempenho de mercado (QTOB). Esse resultado pode ser explicado pela Figura I, na qual se nota que ao longo dos anos as empresas do setor de saúde sofreram diminuição no desempenho operacional, especialmente a partir de 2015, enquanto o desempenho de mercado oscilou sem grandes variações. Como as práticas de responsabilidade social corporativa se mantiveram constantes e o desempenho operacional sofreu uma queda (Figura I), espera-se que haja uma relação negativa, apesar de que tais práticas impulsionam o desempenho empresarial (ABOUD; DIAB, 20I8; BROOKS; OIKONOMOU, 20I8; YU et al., 20I8).

O pilar governança (GOV) reflete (i) o compromisso e a eficácia da empresa em seguir os princípios de governança corporativa de melhores práticas; (ii) a eficácia da empresa em relação à igualdade de tratamento dos acionistas e ao uso de dispositivos antitakeover, e (iii) as práticas da empresa para comunicar que integra as dimensões econômica, social e ambiental em suas decisões no dia a dia (THOMSON REUTERS, 2019).

$\mathrm{Na}$ Tabela 4, o pilar governança (GOV) apresenta resultado significativo no tocante ao desempenho operacional (ROA) e ao desempenho de mercado (QTOB), sendo, contudo, uma relação negativa e positiva, respectivamente, o que corrobora os achados de Buallay (2019) e Li et al. (2018). Ao buscar melhores práticas de governança corporativa, portanto, as empresas do setor de saúde seriam menos lucrativas. Entretanto, como a governança corporativa reduz os conflitos de interesses, as empresas do setor de saúde favorecem os stakeholders, o que se reflete no desempenho de mercado (QTOB), contribuindo para a sua legitimação no mercado. Assim como a responsabilidade social corporativa, a governança corporativa se manteve constante, enquanto o desempenho empresarial operacional (ROA) apresentou uma queda, conduzindo para uma relação negativa.

Como individualmente as práticas ambientais, sociais e de governança apresentaram uma relação negativa com o desempenho operacional (ROA) e uma relação positiva com o desempenho de mercado (QTOB), era esperado que o escore ESG, que representa a ação conjunta da responsabilidade social corporativa com a governança corporativa, apresentaria as mesmas relações.

Desse modo, conforme demonstrado na Tabela 4, observa-se que o escore ESG apresentou uma relação negativa com o desempenho operacional (ROA), portanto, rejeita-se a Hipótese 2. No entanto, 
não se rejeita a Hipótese 3, pois as práticas de responsabilidade social corporativa e governança corporativa acarretam mudanças apenas na percepção dos stakeholders (HOLDER-WEBB et al., 2009), sendo relevante para os investidores (CORAM; MONROE; WOODLIFF, 2009), legitimando a empresa e criando valor de mercado (BARNEA; HEINKEL; KRAUS, 20I3; BROOKS; OIKONOMOU, 20I8; ECCLES; IOANNOU; SERAFEIM, 20I4; FATEMI; GLAUM; KAISER, 20I7; JO; HARJOTO 20I I; NTIM; SOOBAROYEN, 20I3; PEIRIS; EVANS, 20I0).

Quanto às variáveis de controle, observa-se um resultado divergente, a depender da abordagem do desempenho empresarial (ROA e QTOB), com exceção da geração de caixa (FCO), que apresentou correlação positiva com as duas abordagens, condizentemente com Lonial et al. (2008). Em relação ao ROA, as empresas maiores (TAM) (XIE et al., 2019), com baixo crescimento (CRES), menos endividadas (END) (WANG; SARKIS, 20I7), geradoras de caixa (FCO) (LONIAL et al., 2008), e sediadas em países de menor desenvolvimento social (IDH) e econômico (PIB) (BUALLAY, 20I9) detêm maior desempenho operacional (ROA). Em relação ao $Q$ de Tobin, por sua vez, as empresas menores (TAM) (XIE et al., 2019), mais endividadas (END) (WANG; SARKIS, 20I7), geradoras de caixa (FCO) (LONIAL et al., 2008), sediadas em países de maior desenvolvimento social (IDH) e econômico (PIB) detêm maior desempenho de mercado (QTOB).

\section{CONCLUSÃO}

As empresas do setor de saúde se encontram em um ambiente complexo, com alta regulação, relevante para a economia, e têm impacto no bem-estar humano, sendo necessário o equilíbrio entre responsabilidade social corporativa, governança corporativa e desempenho empresarial na busca da sua continuidade e legitimação. Diante do exposto, e considerando-se os preceitos da Teoria da Legitimidade, analisou-se aqui o efeito da governança corporativa e da responsabilidade social corporativa no desempenho empresarial no setor de saúde. Foram examinados dados de 453 empresas de capital aberto do setor de saúde, com sede em 3 I países, referentes a período 20I0-20I8, totalizando I.825 observações.

Os resultados demonstraram que o escore ESG, que representa a responsabilidade social corporativa e a governança corporativa, possui valor mediano e apresenta um comportamento constante, enquanto o desempenho empresarial operacional (mensurado pelo ROA) apresentou queda, e o desempenho empresarial de mercado (representado pelo QTOB) oscilou com leve queda, em meio à melhoria do desenvolvimento econômico e social (PIB, IDH e IGN) dos países.

Por meio da correlação de Pearson, concluiu-se pela não rejeição da Hipótese I, em que, pela Teoria da Legitimidade, a responsabilidade social corporativa e a governança corporativa são positivamente relacionadas e responsáveis pela legitimação da empresa no mercado.

Por meio da regressão quantílica, por sua vez, observou-se que os pilares ambiental, social e de governança são positivamente relacionados com o desempenho empresarial de mercado (QTOB), sendo encontrado um resultado inverso quanto ao desempenho empresarial operacional (ROA). Considerando-se o efeito conjunto da responsabilidade social corporativa com a governança corporativa, mensurado pelo escore ESG, verifica-se o mesmo comportamento, rejeitando-se a Hipótese 2, haja vista a correlação inversa quanto ao desempenho operacional (ROA), e não se rejeitando a Hipótese 3, visto que se verificou um relacionamento positivo entre o desempenho de mercado (QTOB) e o escore ESG.

Constatou-se ainda que o desenvolvimento econômico (PIB) e social (IDH e IGN) do país afeta o desempenho empresarial, a responsabilidade social corporativa e a governança corporativa, podendo contribuir para a legitimação das empresas.

Esses resultados têm implicações práticas para as empresas do setor de saúde, pois se seus gestores almejam garantir a continuidade no mercado e, assim, a sua legitimação, devem se preocupar em atender às exigências dos diversos stakeholders e buscar a redução de conflitos de interesses. Logo, precisam investir em práticas de responsabilidade social corporativa e de governança corporativa, o que pode criar valor. Além disso, o estudo contribui significativamente para enriquecer a literatura 
pertinente, já que não foram identificados estudos sobre as relações entre responsabilidade social corporativa, governança corporativa e desempenho empresarial no setor de saúde.

Destaca-se, por último, a limitação do estudo quanto à amostra, pois foram consideradas apenas as empresas do setor de saúde que possuíam dados da ESG na Thomson Reuters Eikon. Com vistas a pesquisas futuras, sugere-se ampliar a amostra, assim como a utilização de distintos indicadores. Sugerese ainda analisar o disclosure da responsabilidade social corporativa e da governança corporativa das empresas, bem como o efeito de tais práticas e do desempenho empresarial no gerenciamento de resultados.

\section{REFERENCIAS}

ABOUD, A.; DIAB, A. The impact of social, environmental and corporate governance disclosures on firm value: evidence from Egypt. Journal of Accounting in Emerging Economies, v. 8, n.4, 442-458, 2018.

AGUILERA, R. V.; RUPP, D. E.; WILLIAMS, C. A.; GANAPATHI, J. Putting the S back in corporate social responsibility: a multilevel theory of social change in organizations. Academy of Management Review, v. 32, n. 3, 836-863, 2007.

ANDRIOF, J.; WADDOCK, S. Unfolding stakeholder thinking. In J. Andriof, S. Waddock, B. Husted, \& Rahman, S. (Eds.): Unfolding stakeholder thinking: theory, responsibility, and enlightenment, 19-42, Greenleaf, Sheffield, London: Routledge, 2002.

BARNEA, A.; HEINKEL, R.; KRAUS, A. Corporate social responsibility, stock prices and tax policy. Canadian Journal of Economics. v. 46, n. 3, I066-1084, 2013.

BELTRATTI, A. The complementarity between corporate governance and corporate social responsibility. The Geneva Papers on Risk and Insurance-Issues and Practice, v. 30, n. 3, 373-386, 2005.

BENNINGTON, L. Review of the corporate and healthcare governance literature. Journal of Management \& Organization, v. I6, n. 2, 3I4-333, 2010.

BROOKS, C.; OIKONOMOU, I. The effects of environmental, social and governance disclosures and performance on firm value: a review of the literature in accounting and finance. The British Accounting Review, v. 50, n. I, I-I5, 2018.

BUALLAY, A. Is sustainability reporting (ESG) associated with performance? Evidence from the European banking sector. Management of Environmental Quality: An International Journal, v. 30, n. I, 98-II5, 2019.

BURKE, E. Managing a company in an activist world: the leadership challenge of corporate citizenship, Praeger, Westport, CT, London: Praeger, 2005.

CADBURY, S. A. Thoughts on corporate governance. Corporate Governance: An International Review, v. I, 5-I0, 1993.

CHAMBERS, N. Healthcare board governance. Journal of Health Organization and Management, v. 26, n. I, 6-I4, 2012. 
CHAN, M. C.; WATSON, J.; WOODLIFF, D. Corporate governance quality and CSR disclosures. Journal of Business Ethics, v. I25, n. I, 59-73, 2014.

CHEN, V. Z.; LI, J.; SHAPIRO, D. M. Are OECD-prescribed "good corporate governance practices" really good in an emerging economy? Asia Pacific Journal of Management, v. 28, n. I, I I5-I38, $201 \mathrm{I}$.

CORAM, P. J.; MONROE, G. S.; WOODLIFF, D. R. The value of assurance on voluntary nonfinancial disclosures: an experimental evaluation. Auditing: A Journal of Practice and Theory, v. 28, n. I, I37-I5I, 2009.

CRIFO, P.; DIAYE, M. A.; OUEGHLISSI, R. The effect of countries' ESG ratings on their sovereign borrowing costs. The Quarterly Review of Economics and Finance, v. 66, I3-20, 2017.

CUERVO, A. Corporate governance mechanisms: a plea for less code of good governance and more market control. Corporate Governance: An International Review, v. I0, n. 2, 84-93, 2002.

DEEGAN, C. The legitimising effect of social and environmental disclosures - a theoretical foundation. Accounting, Auditing and Accountability Journal, v. I5, n. 3, 282-3I I, 2002.

DEL BOSCO, B.; MISANI, N. The effect of cross-listing on the environmental, social, and governance performance of firms. Journal of World Business, v. 5I, n. 6, 977-990, 2016.

DOWLING, J.; PFEFFER, J. Organizational legitimacy: social values and organizational behavior. Pacific Sociological Review, v. I8, n. I, I22-I36, I975.

ECCLES, R. G.; IOANNOU, I.; SERAFEIM, G. The impact of corporate sustainability on organizational processes and performance. Management Science, v. 60, n. II, 2835-2857, 2014.

EECKLOO, K.; HERCK, G.; HULLE, C.; VLEUGELS, A. From corporate governance to hospital governance: authority, transparency and accountability of Belgian non-profit hospitals' board and management. Health Policy, v. 68, n. I, I-I5, 2004.

ELLIOTT, W. B.; JACKSON, K. E.; PEECHER, M. E.; WHITE, B. J. The unintended effect of corporate social responsibility performance on investors' estimates of fundamental value. The Accounting Review (January), v. 89, n. I, 275-302, 2014.

FATEMI, A.; GLAUM, M.; KAISER, S. ESG performance and firm value: the moderating role of disclosure. Global Finance Journal, v. 38, 45-64, 2017.

FÁVERO, L. P.; BELFIORE, P. Manual de análise de dados: estatística e modelagem multivariada com

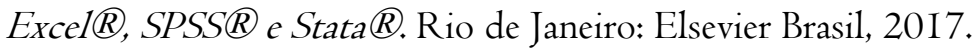

FOMBRUN, C.; SHANLEY, M. 'What's in a name? Reputation building and corporate strategy'. Academy of Management Journal, v. 33, n. 2, 233-258, 1990.

FRIEDMAN, M. "The social responsibility of business is to increase its profits". New York Times Magazine, I3 September (32-33), I22-I24, I970.

GARCIA, A. S.; MENDES-DA-SILVA, W.; ORSATO, R. J. Sensitive industries produce better ESG performance: evidence from emerging markets. Journal of Cleaner Production, v. I50, I35-I47, 2017. 
GEIGER, S. W.; MARLIN, D.; SEGREST, S. L. Slack and performance in the hospital industry: a configurational approach. Management Decision, v. 57, I-20, 2019.

HART, O. Corporate governance: some theory and implications. The Economic Journal, v. I05, n. 430, 678-689, I995.

HART, S. L. Capitalism at the crossroads: next generation business strategies. Upper Saddle River, NJ: Prentice Hall, 2010.

HART, S. L.; MILSTEIN, M. Creating sustainable value. Academy of Management Executive, v. I7, n. 2, 56-69, 2003.

HOLDER-WEBB, L.; COHEN, J.; NATH, L.; WOOD, D. The supply of corporate social responsibility disclosure among U. S. firms. Journal of Business Ethics (February), v. 84, n. 4, 497-527, 2009.

IOANNOU, I.; SERAFEIM, G. What drives corporate social performance? The role of nation-level institutions. Journal of International Business Studies, v. 43, n. 9, 834-864, 2012.

JAMALI, D.; HALLAL, M.; ABDALLAH, H. Corporate governance and corporate social responsibility: evidence from the healthcare sector. Corporate Governance: The International Journal of Business in Society, v. I0, n. 5, 590-602, 2010.

JO, H.; HARJOTO, M. A. Corporate governance and firm value: the impact of corporate social responsibility. Journal of Business Ethics, v. I03, n. 3, 35I-383, 201 I.

KAYMAK, T.; BEKTAS, E. Corporate social responsibility and governance: information disclosure in multinational corporations. Corporate Social Responsibility and Environmental Management, v. 6, n. 24, 555-569, 2017.

KAUFMANN, D.; KRAAY, A.; ZOIDO-LOBATÓN, P. Aggregating governance indicators (Vol. 2I95). Washington, DC: World Bank Publications, 1999.

LA PORTA, R.; LÓPEZ-DE-SILANES, F.; SHLEIFER, A.; VISHNY, R. Law and finance, Journal of Political Economy, v. I06, n. 6, III3-I II5, 1998.

LERACH, W. S. Plundering America: how American investors got taken for trillions by corporate insiders. Stanford Journal of Law, Business and Finance, v. 8, 69-I28, 2002.

LI, Y.; GONG, M.; ZHANG, X.; KOH, L. The impact of environmental, social, and governance disclosure on firm value: the role of CEO power. The British Accounting Review, v. 50, n. I, 60-75, 2018.

LIOUI, A.; SHARMA, Z. Environmental corporate social responsibility and financial performance: Disentangling direct and indirect effects. Ecological Economics, v. 78, I00-I I I, 2012.

LONIAL, S. C.; TARIM, M.; TATOGLU, E.; ZAIM, S.; ZAIM, H. The impact of market orientation on NSD and financial performance of hospital industry. Industrial Management \& Data Systems, v. I08, n. 6, 794-8I I, 2008. 
MARGARETIC, P.; POUGET, S. Sovereign bond spreads and extra-financial performance: An empirical analysis of emerging markets. International Review of Economics \& Finance, v. 58, 340-355, 2018.

MASON, C.; SIMMONS, J. Embedding corporate social responsibility in corporate governance: a stakeholder systems approach. Journal of Business Ethics, v. I I9, n. I, 77-86, 2014.

MCBARNET, D.; VOICUlESCU, A.; CAMPBELL, T. The New Corporate Accountability: Corporate Social Responsibility and the Law, Cambridge University Press, Cambridge, 2007.

MCWILLIAMS, A.; SIEGEL, D. Corporate social responsibility and financial performance: Correlation or misspecification?, Strategic Management Journal, v. 2I, 603-609, 2000.

MICHELON, G.; PARBONETTI, A. The effect of corporate governance on sustainability disclosure. Journal of Management and Governance, v. I6, n. 3, 477-509, 2012.

MONEY, K.; SCHEPERS, H. Are CSR and corporate governance converging? A view from boardroom directors and company secretaries in FTSEIOO companies in the UK. Journal of General Management, v. 33, n. 2, I-II, 2007.

MONFARDINI, P.; BARRETTA, A. D.; RUGGIERO, P. Seeking legitimacy: Social reporting in the healthcare sector. Accounting Forum, v. 37, n. I, 54-66, 2013.

NTIM, C. G.; SOOBAROYEN, T. Corporate governance and performance in socially responsible corporations: New empirical insights from a Neo-Institutional framework. Corporate Governance: An International Review, v. 2I, n. 5, 468-494, 2013.

PEIRIS, D.; EVANS, J. The relationship between environmental social governance factors and US stock performance. Joumal of Investing, v. I9, n. 3, I04-I I2, 20 I0.

PLUMLEE, M.; BROWN, D.; HAYES, R. M.; MARSHALL, R. S. Voluntary Environmental Disclosure Quality and Firm Value: Further Evidence. Journal of Accounting and Public Policy, v. 34, n. 4, 336-36I, 2015.

POINTER, D. D.; ORLIKOFF, J. E. Board work: Governing health care organizations. San Francisco: Jossey-Bass, I999.

PORTER, M.; KRAMER, M. Creating shared value. Harvard Business Review, v. 89, n. I/2, 62-77, $20 I I$.

REAZ, M.; HOSSAIN, M. Corporate governance around the world: an investigation. Journal of American Academy of Business, v. II, n. 2, I69-175, 2007.

REINHARDT, F.; STAVINS, R.; VIETOR, R. Corporate social responsibility through an economic lens. Review of Environmental Economics and Policy, v. 2, n. 2, 219-239, 2008.

SCOTT, W. R. Financial accounting theory. Ontario: Pearson, 2015.

SHLEIFER, A.; VISHNY, R. W. A survey of corporate governance. The Journal of Finance, v. 52, n. 2, 737-783, I997. 
STEINGRABER, F. G.; KANE, K. What boards need to do to preserve their relevance and provide value in the world of the new normal. Corporate Finance Review, v. I5, n. I, I-6, 2010.

SUCHMAN, M. C. Managing legitimacy: strategic and institutional approaches. Academy of Management Review, v. 20, n. 3, 57I-6I0, I995.

THOMSON REUTERS. Thomson Reuters ESG scores, 2019. Retrieved from https://www.refinitiv.com/content/dam/marketing/en_us/documents/methodology/esg-scoresmethodology.pdf

TIME MAGAZINE. 4 new truths from the stock market crisis of 2015, 2015. Retrieved from https://time.com/4008762/stock-market/

TRICKER, B. Corporate governance: principles, policies, and practices, Oxford, UK: Oxford University Press, 2009.

WALLS, J. L.; BERRONE, P.; PHAN, P. H. Corporate governance and environmental performance: is there really a link? Strategic Management Journal, v. 33, n. 8, 885-9I3, 2012.

WANG, Z.; SARKIS, J. Corporate social responsibility governance, outcomes, and financial performance. Journal of Cleaner Production, v. I62, n. 20, I607-I6I6, 2017.

WICKRAMASINGHE, N. Encyclopedia of healthcare information systems. New York: IGI Global, 2008.

XIE, J.; NOZAWA, W.; YAGI, M.; FUJII, H.; MANAGI, S. Do environmental, social, and governance activities improve corporate financial performance?, Business Strategy and the Environment, v. 28, n. 2, 286-300, 2019.

YU, E. P. Y.; GUO, C. Q.; LUU, B. V. Environmental, social and governance transparency and firm value. Business Strategy and the Environment, v. 27, n. 7, 987-1004, 2018.

YU, T. H.; WANG, D. H.; WU, K. Reexamining the red herring effect on healthcare expenditures. Journal of Business Research, v. 68, n. 4, 783-787, 2015. 